Proc. Indian Acad. Sci. (Chem. Sci.), Vol. 105, No. 6, December 1993, pp. 333-342.

(C) Printed in India.

\title{
Photobiotechnology: Application of photosynthesis to the production of renewable fuels and chemicals
}

\author{
E GREENBAUM \\ Chemical Technology Division, Oak Ridge National Laboratory, P. O. Box 2008, Oak Ridge, \\ TN 37831-6194, USA
}

\begin{abstract}
Sustained hydrogen photoevolution from Chlamydomonas reinhardtii and $C$. moewusii was measured under an anoxic, $\mathrm{CO}_{2}$-containing atmosphere. It has been discovered that light intensity-and temperature influence the partitioning of reductant between the hydrogen photoevolution pathway and the Calvin cycle. Under low incident light intensity $\left(1-3 \mathrm{~W} \mathrm{~m}^{-2}\right)$ or low temperature $\left(\approx 0^{\circ} \mathrm{C}\right)$, the flow of photosynthetic reductant to the Calvin cycle was reduced, and reductant was partitioned to the hydrogen pathway as evidenced by sustained $\mathrm{H}_{2}$ photoevolution. Under saturating light $\left(25 \mathrm{~W} \mathrm{~m}^{-2}\right)$ and moderate temperature $\left(20 \pm 5^{\circ} \mathrm{C}\right)$, the Calvin cycle became the absolute sink for reductant with the exception of a burst of $\mathrm{H}_{2}$ occurring at light on. A novel photobiophysical phenomenon was observed in isolated spinach chloroplasts that were metalized by precipitating colloidal platinum onto the surface of the thylakoid membranes. A two-point irradiation and detection system was constructed in which a continuous beam helium-neon laser $(\lambda=632.8 \mathrm{~nm})$ was used to irradiate the platinized chloroplasts at varying perpendicular distances from a single linear platinum electrode in pressure contact with the platinized chloroplasts. No external voltage bias was applied to the system. The key objective of the experiments reported in this report was to measure the relative photoconductivity of the chloroplast-metal composite matrix.
\end{abstract}

Keywords. Photobiotechnology; photosynthesis; hydrogen; oxygen; platinized chloroplasts; water splitting.

\section{Introduction}

\subsection{Hydrogen production}

Hydrogen photoevolution by unicellular algae was first demonstrated by Gaffron and Rubin (1942). Since their pioneering discovery, the reducing power driving sustained hydrogen photoevolution under anaerobiosis has been shown to be primarily derived from the water-splitting reaction of photosystem II (Bishop et al 1977). In green algae, an oxygen-labile hydrogenase couples the oxidation of reduced ferredoxin with hydrogen evolution. Hydrogenase is a critical catalyst for a number

\footnotetext{
'The submitted manuscript has been authored by a contractor of the US Government under contract No. DE-AC05-840R21400. Accordingly, the US Government retains a nonexclusive, royalty-free license to publish or reproduce the published form of this contribution, or allow others to do so, for US Government purposes.'
} 
of single and multistep reactions. Experimental conditions determine which reactions proceed (Bishop et al 1977; Senger and Bishop 1979).

Light activated:

(1) Photoreduction: $\mathrm{CO}_{2}+2 \mathrm{H}_{2}+$ light $\rightarrow \mathrm{CH}_{2} \mathrm{O}+\mathrm{H}_{2} \mathrm{O}$,

(2) $\mathrm{H}_{2}$ photoproduction: $\mathrm{XH}_{2}+$ light $\rightarrow \mathrm{X}+\mathrm{H}_{2}$.

Thermally activated:

(3) Oxy-hydrogen reaction: $2 \mathrm{H}_{2}+\mathrm{O}_{2} \rightarrow 2 \mathrm{H}_{2} \mathrm{O}$,

(4) Dark $\mathrm{CO}_{2}$ fixation: $\mathrm{CO}_{2}+\mathrm{H}_{2}+$ energy $\rightarrow \mathrm{CH}_{2} \mathrm{O}+\mathrm{H}_{2} \mathrm{O}$,

(5) $\mathrm{H}_{2}$ production: $\mathrm{RH}_{2} \rightarrow \mathrm{R}+\mathrm{H}_{2}$,

(6) $\mathrm{H}_{2}$ uptake: $\mathrm{R}+\mathrm{H}_{2} \rightarrow 2 \mathrm{H}_{2}$.

In the absence of $\mathrm{CO}_{2}$, the absolute light-saturated rate of electron transport is greatly reduced. Under these experimental conditions hydrogen evolution is the main pathway for the utilization of photoproduced reductant. The steady-state ratio for $\mathrm{H}_{2}$ to $\mathrm{O}_{2}$ evolution is usually close to two, indicating that essentially all electrons generated by the biophotolysis of water are expressed as $\mathrm{H}_{2}$ (Greenbaum 1984; Ward et al 1985). If $\mathrm{CO}_{2}$ is available sustained hydrogen photoproduction does not occur (Gaffro.l and Rubin 1942; Stuart and Gaffron 1972). Gaffron and Rubin (1942) demonstrated that under anaerobic conditions dinitrophenol inhibited $\mathrm{CO}_{2}$ reduction in Scenedesmus and allowed hydrogen photoproduction to occur in the presence of $\mathrm{CO}_{2}$. Similarly, glucose and carbonyl cyanide- $m$-chlorophenylhydrazone stimulated $\mathrm{H}_{2}$ photoevolution in the presence of $\mathrm{CO}_{2}$ (Kaltwasser et al 1969). Those experiments suggested the coexistence of two reductant sinks, with the Calvin cycle being the primary sink in the presence of $\mathrm{CO}_{2}$ and the hydrogen pathway serving as an alternate sink that functioned when $\mathrm{CO}_{2}$ was absent or when the activity of the Calvin cycle was inhibited.

Stuart and Gaffron (1972) approached the problem of dual, competitive pathways using a closed system and chemical inhibitors. However, a closed-system approach is severely limited in the useful information that can be obtained. For example, uniform conditions are impossible to maintain for the duration of an experiment because the organisms modify their environment by liberating $\mathrm{O}_{2}$ and $\mathrm{H}_{2}$ and consuming $\mathrm{CO}_{2}$. This situation leads to non-steady-state conditions that stimulate several of the aforementioned hydrogenase mediated reactions, thereby greatly complicating the interpretation of experimental results. The experiments described in this review have taken a new approach to this problem. First, continuous gas flow was used; this prevented $\mathrm{O}_{2}$ inactivation of hydrogenase resulting from the accumulation of $\mathrm{O}_{2}$, which occurred in closed systems such as those used in most other studies of anaerobic photosynthesis. Second, continuous flow also removed photoproduced $\mathrm{H}_{2}$ and $\mathrm{O}_{2}$ so that the oxy-hydrogen and photoreduction reactions, photorespiration, and respiration did not occur. Third, the concentration of $\mathrm{CO}_{2}$ was maintained at a constant level by bubbling an $\mathrm{He}-\mathrm{CO}_{2}$ gas mixture through the sample instead of supplying $\mathrm{CO}_{2}$ from finite bicarbonate or gas phase source (Gaffron and Rubin 1942; Healey 1970; Stuart and Gaffron 1972; Gfeller and Gibbs 1985). Fourth, the ability to perform long-term experiments in which all measured reactions were driven into steady-state provided additional information not available previously. The coexistence of two pathways that directly utilize photogenerated reductant provides a unique opportunity to experimentally examine the kinetic competition between the pathways for reductant and the regulatory mechanisms that work to control and balance the 
activities of the Calvin cycle, the hydrogen pathway, and the photosynthesis. The key discovery of this report is control of partitioning of photogenerated reductant between the hydrogen pathway and the Calvin cycle, by variable light intensity and temperature.

\subsection{Photoconductivity}

Photosynthesis is vectorial photochemistry. Light quanta that are captured in photosynthetic reaction centers initiate a primary electron transfer reaction, resulting in spatial separation of electrostatic charge across the photosynthetic membrane. The vectorial nature of photosynthesis lies in the intrinsic orientation of the reaction centers embedded in the membranes. Electron flow is from the inner membrane surface of the flattened sac-like vesicles to the outer surface (Amesz 1987). In normal photosynthesis, electrons from the reducing end of photosystem I are used for the enzymatic reduction of $\mathrm{NADP}^{+}$to NADPH. This reduction is mediated by ferredoxin and ferredoxin NADP-reductase. NADPH serves as the electron carrier to the Calvin cycle for the enzymatic reduction of atmospheric carbon dioxide to plant matter.

It has been shown that colloidal platinum can be precipitated onto the surface of photosynthetic membranes so that water is photobiocatalytically split into molecular hydrogen and oxygen upon illumination with light of any wavelength in the chlorophyll absorption spectrum (Greenbaum 1985). Since no electron mediator such as ferredoxin or methyl viologen was present, the colloidal platinum must have been precipitated sufficiently close to the photosystem I reduction site to allow interfacial electron transfer from the membrane to the platinum. The presence of a reticulated network of platinum particles embedded in the chloroplast matrix suggests that metal-like properties can, at least partially, be imparted to the chloroplasts. In this work, the relative photoconductivity of the material was measured by the flow of photocurrent in the plane of the entrapped platinized chloroplasts of uniform thickness from the point of laser irradiation to the linear platinum wire electrode.

\section{Results}

\subsection{Hydrogen production}

Under anaerobic conditions in the absence of $\mathrm{CO}_{2}$, the ratio of light-saturated, steady-state $\mathrm{H}_{2}$ and $\mathrm{O}_{2}$ photoevolution by $C$. reinhardtii approached two. However, when $\mathrm{CO}_{2}$ was introduced, photosaturated $\mathrm{O}_{2}$ evolution increased dramatically and light-driven $\mathrm{H}_{2}$ evolution ceased, except for a transient burst that occurred when the light was turned on. These observations were consistent with those of Stuart and Gaffron (1972).

The 5-10-fold increase in light-driven $\mathrm{O}_{2}$ production corresponded with the typical increase attributed to the bicarbonate effect on PS II activity (Vermaas and Govindjee 1981). The lack of sustained $\mathrm{H}_{2}$ photoevolution suggested that $\mathrm{CO}_{2}$ also affected the activation of the Calvin cycle because $\mathrm{CO}_{2}$ is well known to activate RuBP Carboxylase by providing substrate and positive effector (Lorimer et al 1976). The Calvin cycle provided a faster kinetic sink for reductant than the $\mathrm{H}_{2}$ pathway thereby eliminating $\mathrm{H}_{2}$ photoevolution under conditions where photosynthesis and the Calvin cycle were fully activated. 


\subsection{Hydrogenase activity in the presence of $\mathrm{CO}_{2}$}

Dark hydrogen evolution and the burst of $\mathrm{H}_{2}$ accompanying 'light on' in the presence of $\mathrm{CO}_{2}$ indicated that hydrogenase activity was not adversely affected by $\mathrm{CO}_{2}$ or the high $\mathrm{O}_{2}$ photoevolution rate in the continuous flow experimental system. The yield of $\mathrm{H}_{2}$ represented by the burst at 'light on' was about $25 \mathrm{nmol}$ for the cell suspension containing $50 \mu \mathrm{g} \mathrm{Chl}$. Normalizing the data to $1 \mathrm{mg}$ of $\mathrm{Chl}$, the $\mathrm{H}_{2}$ yield from the burst was $500 \mathrm{nmol} / \mathrm{mg} \mathrm{Chl}$. By measuring the $\mathrm{H}_{2}$ yield associated with the burst in other, similar experiments, the yield was found to be variable, ranging from 200 to $1000 \mathrm{nmol} / \mathrm{mg} \mathrm{Chl}$ (data not shown). This information allowed an estimation of the number of reducing equivalents contributed by each photosynthetic electron transport chain for the transient burst. The following calculation was based on the data normalized to $1 \mathrm{mg} \mathrm{Chl}$.

$500 \times 10^{-7} \mathrm{~mol} \mathrm{H}_{2} /\left(1.1 \times 10^{-6} \mathrm{~mol} \mathrm{Chl} / 500 \mathrm{~mol} \mathrm{Chl} / \mathrm{mol}\right.$ photosynthetic electron transport chains) $=227 \mathrm{~mol} \mathrm{H}_{2} / \mathrm{mol}$ electron transport chain.

With 2 electrons per $\mathrm{H}_{2}$, a total of 454 electrons were spent in the burst of $\mathrm{H}_{2}$. This number far exceeds $\approx 5-20$ reducing equivalents per electron transport chain stored in a completely reduced plastoquinone pool (Joliot 1965; Forbush and Kok 1968; Stiehl and Witt 1969; Greenbaum 1979).

To determine the source of the reductant driving the burst of $\mathrm{H}_{2}$ at light-on in the presence of $\mathrm{CO}_{2}$, an ethanolic solution of DCMU was added to the algal cells to give a final DCMU concentration of $15 \mu \mathrm{M}$. This level of DCMU completely eliminated the burst of $\mathrm{H}_{2}$ at light-on as well as $\mathrm{O}_{2}$ photoevolution (data not shown). However, sustained light-driven $\mathrm{H}_{2}$ evolution was observed for a few hours after the addition of DCMU demonstrating that stored reductant can be used for light-driven $\mathrm{H}_{2}$ photoevolution.

\subsection{The effect of light intensity on $\mathrm{H}_{2}$ and $\mathrm{O}_{2}$ photoevolution}

Light intensity was varied from 0 to $25 \mathrm{~W} \mathrm{~m}^{-2}$. Under low-light conditions, sustained $\mathrm{H}_{2}$ photoevolution was detected with the maximum ratio of $\mathrm{H}_{2}$ to $\mathrm{O}_{2}$ being about one at $0^{\circ} \mathrm{C}$, sustained, albeit low, $\mathrm{H}_{2}$ photoproduction was observed even at the highest light intensity.

In the absence of $\mathrm{CO}_{2}$ at $20^{\circ} \mathrm{C}$ and at various light intensities, $\mathrm{H}_{2}$ and $\mathrm{O}_{2}$ were coevolved with a hydrogen-to-oxygen ratio of about 2 , as previously reported (Greenbaum 1984). The yield of $\mathrm{H}_{2}$ and $\mathrm{O}_{2}$ at $0^{\circ} \mathrm{C}$ was similar to that seen at $20^{\circ} \mathrm{C}$, and the stoichiometry of $\mathrm{H}_{2}$-to- $\mathrm{O}_{2}$ was $\approx 2$.

\subsection{The effect of temperature on $\mathrm{H}_{2}$ and $\mathrm{O}_{2}$}

Light-driven $\mathrm{H}_{2}$ production was measured at temperatures ranging from 0 to $40^{\circ} \mathrm{C}$. Pretreatment of the algae had a significant effect on $\mathrm{O}_{2}$ and $\mathrm{H}_{2}$ photoevolution. The cells adapted to changes in temperature by shifting their optimal photoproduction temperature. For example, cells held overnight at $0^{\circ} \mathrm{C}$ showed higher $\mathrm{O}_{2}$ yields at $5^{\circ} \mathrm{C}$ than cells taken from 20 to $5^{\circ} \mathrm{C}$ within $2 \mathrm{~h}$ and assayed immediately. After cooling to $0^{\circ} \mathrm{C}$, the algae were assayed for $1 \mathrm{~h}$ then the temperature was raised by $5^{\circ} \mathrm{C}$ and the same algae were assayed for $1 \mathrm{~h}$ followed by another $5^{\circ} \mathrm{C}$ temperature adjustment 
and $1 \mathrm{~h}$ assay until $40^{\circ} \mathrm{C}$ was reached. The temperature changes required less than $5 \mathrm{~min}$ each and the steady-state rate of photoevolution was then measured after $1 \mathrm{~h}$ of irradiation.

At $0^{\circ} \mathrm{C}$ steady-state $\mathrm{H}_{2}$ and $\mathrm{O}_{2}$ photoevolution occurred in the presence of $\mathrm{CO}_{2}$. The ratio of $\mathrm{H}_{2}$ to $\mathrm{O}_{2}$ was about 1.2 , indicating that the hydrogen pathway was not the sole pathway for reductant utilization. Under these conditions, competition for reductant evidently existed between the Calvin cycle and the $\mathrm{H}_{2}$ pathway. Moderate temperatures $\left(>5\right.$ and $<30^{\circ} \mathrm{C}$ ) caused the cessation of $\mathrm{H}_{2}$ photoevolution; however, at warmer temperatures $\left(>30^{\circ} \mathrm{C}\right)$, a small portion of the photogenerated reductant was shunted to the hydrogen pathway.

In the absence of $\mathrm{CO}_{2}$, the $\mathrm{H}_{2}$-to- $\mathrm{O}_{2}$ photoevolution ratio remained close to 2 until the temperature rose to $>25^{\circ} \mathrm{C}$. At warmer temperatures, the $\mathrm{H}_{2}-$ to- $\mathrm{O}_{2}$ ratio significantly exceeded 2 , suggesting that additional reductant was being supplied from a source other than PS II. The alternative source, which may enter the photosynthetic electron transport chain through the plastoquinone pool (Diner and Mauzerall 1973; Gfeller and Gibbs 1985; Peltier et al 1987), was light driven since $\mathrm{H}_{2}$ photoevolution fell to zero when the light was turned off.

\subsection{Photoconductivity}

Table 1 presents data for four platinum concentrations. A 5-ml suspension of spinach chloroplasts (containing $3 \mathrm{mg}$ of chlorophyll) was used. Platinization of chloroplasts is feasible because hexachloroplatinate can be converted to metallic platinum at $\mathrm{pH} 7$ and room temperature (Anderson 1975). These are experimental conditions that preserve photoactivity of the isolated chloroplasts. The platinized chloroplasts were entrapped on a thin, fiberglass filter pad (Millipore, AP40) and were moistened with Walker's assay medium (Walker 1980), in which the chloroplasts were suspended. The fiberglass filter pad was $0.3 \mathrm{~mm}$ thick, had an active filtration area of $10.4 \mathrm{~cm}^{2}$, and contained no binder resins. The thickness of the chloroplast film was estimated to be between 0.01 and $0.1 \mathrm{~mm}$. A silver-silver chloride reference electrode was placed

Table 1. Vectorial photocurrents and electron-transfer distances in platinized chloroplasts as a function of platinum loading.

\begin{tabular}{lccc}
\hline Line no. & $I_{0},{ }^{2} \mathrm{nA}$ & $D_{\max }, \mathrm{mm}$ & {$[\mathrm{Pt}]^{\mathrm{b}}, \mathrm{mg} / \mathrm{ml}$} \\
\hline 1 & 0 & 0 & 0.24 \\
2 & 0 & 0 & 0.49 \\
3 & 3.6 & 2.3 & 0.97 \\
4 & 7.8 & 3.4 & 1.94
\end{tabular}

\footnotetext{
" $I_{0}$ is defined as the measured photocurrent when $D=0$. This corresponded to the laser beam position when it was directly over the linear electrode and was the maximum current measured in each run.

${ }^{b}$ Aqueous-phase platinum concentration from which the platinum precipitation step was performed. Lines 1 and 2 are the average of two runs each. Line 3 is the average of three runs. Line 4 is the average of six runs.
} 
in pressure contact with the filter; a straight, single, platinum wire of $0.2 \mathrm{~mm}$ diameter was placed in pressure contact with the entrapped platinized chloroplasts. The electrodes, platinized chloroplasts, and filter paper were held together with lucite plates and compression screws. To prevent the electrochemistry of atmospheric oxygen from interfering with the cathode reactions by providing an alternative electron pathway, the entire assembly was placed in a small glass chamber sealed with an $\mathrm{O}$-ring. The $\mathrm{O}$-ring was pierced to allow the passage of two narrow-diameter wires for establishing electrical contact with the electrodes. Premoistened helium gas flowed through the chamber to flush out atmospheric oxygen. After about $45 \mathrm{~min}$, the oxygen concentration of the chamber was below $3 \mathrm{ppm}$ as measured by a calibrated Hersch electrogalvanic cell. Calibration was achieved with an in-line electrolysis cell and Faraday's law of electrochemical equivalence.

The platinum precipitation step was performed in a water-jacketed reactor cell containing $8.0 \mathrm{ml}$ of suspension maintained at $20^{\circ} \mathrm{C}$. Molecular hydrogen was passed over the head space of the reactor while a teflon-coated magnetic stirrer was used to gently stir the chloroplast suspension in a neutral hexachloroplatinate solution. Purge times of 30 to $60 \mathrm{~min}$ were used. After incubation and precipitation, the reactor chamber was opened to air, the contents were filtered onto the filter pad, and the cell was assembled as described previously. This coprecipitation step was essential.

The novel observation in this research is the effect of the precipitated platinum on the photoconductivity of the chloroplast matrix. Each data point represented a steadystate flow of current. Although steady-state was achieved within a few minutes of each change in the laser beam position, sustained photocurrent could be observed for hours. Each data point represents a dwell time of 15 to $20 \mathrm{~min}$.

The photocurrent had a maximum value when the laser beam was directly over the platinum wire. Although the laser beam was partially blocked by the wire electrode when the beam was positioned directly over it, this loss of light was not sufficient to cause a drop in photocurrent. This observation is reasonable since the diameter of the laser beam is larger than the diameter of the platinum wire electrode. Also, the close physical proximity of the laser light and wire on the umbral periphery of the wire provided for efficient charge collection. The extent to which the laser beam could be moved from the wire and still generate measurable photocurrent depended on the concentration of the solution from which the platinum was precipitated. Table 1 is a summary of the data of initial currents and maximum distances that were observed for varying distances.

\section{Discussion}

\subsection{Hydrogen production}

These results establish the coexistence of two competitive pathways for photosynthetically generated reductant and support the findings of Gaffron and coworkers that under anaerobiosis and $\mathrm{CO}_{2}$, inhibitors of photophosphorylation and carbon reduction stimulate $\mathrm{H}_{2}$ photoevolution in microalgae. Light and temperature physically accomplished a result similar to that achieved by the chemical inhibitors used in prior research (Gaffron and Rubin 1942; Stuart and Gaffron 1972). Under low light intensities the activity of many Calvin cycle enzymes is known to be reduced 
(Buchanan 1980), resulting in an imbalance between the number of reducing equivalents generated by photosynthetic water splitting and the number consumed for carbon reduction. At least part of the excess was expressed as $\mathbf{H}_{2}$ via the hydrogen pathway. Similarly, both high and low temperature caused a disparity between production and demand, again leading to the expression of $\mathrm{H}_{2}$. Based on these observations and those of Gaffron and colleagues (Gaffron and Rubin 1942; Stuart and Gaffron 1972), any event that selectively impairs the demand of the Calvin cycle for reductant should lead to the expression of the excess reducing equivalents as $\mathbf{H}_{2}$. The experimental conditions lead to a relative imbalance between reductant demand by the Calvin cycle and reductant supply by photosynthetic water splitting. The results suggest that the activity of the photosynthetic electron transport chain and the photosystems was less sensitive to low temperatures and light regulation than the $\mathrm{Calvin}$ cycle. The fact that $\mathrm{H}_{2}$ photoevolution rates under $\mathrm{He}$ were comparable at both 20 and $0^{\circ} \mathrm{C}$ indicated that hydrogenase was not limiting at the lower temperature.

Hydrogen evolution in the dark was accounted for by the dark hydrogen production reaction (Healey 1970). The burst of $\mathrm{H}_{2}$ at light-on represented $\mathrm{H}_{2}$ photoevolution. The reductant expended in the burst may be from at least three different sources: (1) electrons may be released from the completely reduced plastoquinone pool. One complete release would account for about 10 hydrogen molecules per photosynthetic electron transport chain, assuming $20 \mathrm{PQ} /$ pool. (2) Electrons may be released from a stored pool of reductant other than the plastoquinone pool although this reductant may enter the photosynthetic electron transport chain via the plastoquinone pool (Diner and Mauzerall 1973; Greenbaum 1984; Gfeller and Gibbs 1985; Peltier et al 1987). (3) Hydrogen photoproduction may be directly driven by the biophotolysis of water. Since the amount of reductant necessary to give a hydrogen burst of the measured magnitude must equal about 450 electrons, the plastoquinone pool cannot be the sole source of reductant. An alternative pool, biophotolysis of water, or both may contribute to the transient burst. Inhibition of $\mathrm{O}_{2}$ evolution with DCMU eliminated the burst of $\mathrm{H}_{2}$ at light-on indicating that the burst was driven by the biophotolysis of water. However, the observation of sustained light-driven $\mathrm{H}_{2}$ production in the absence of $\mathrm{O}_{2}$ photoevolution indicated that stored reductant was entering the photosynthetic electron transport system under these conditions. The most likely explanation for the burst of $\mathrm{H}_{2}$ upon illumination is a time delay between photosynthetic reductant production and activation of light regulated Calvin cycle enzymes. During the lag time, reductant is partitioned to the $\mathrm{H}_{2}$ pathway. However, the possibility that electrons from an alternative source contribute to the burst cannot be completely excluded since $\mathrm{H}_{2}$ evolution was observed in the presence of DCMU.

In conclusion, the coexistence of two competitive pathways for photogenerated reducing equivalents has been demonstrated in $C$. reinhardtii and $C$. moewusii adapted for $\mathrm{H}_{2}$ evolution in the presence of $\mathrm{CO}_{2}$. The experimental manipulation of electron partitioning between the two pathways, using the physical parameters of light and temperature, indicates that the pathways coexist under certain experimental conditions. These pathways provide the organisms with a mechanism to avoid becoming 'over reduced' under circumstances where reductant availability exceeds demand. These experiments also demonstrate that the flow of photogenerated reductant can be partially and reversibly switched from carbon fixation to hydrogen evolution for the production of a high-energy, inorganic compound by simply changing conditions in the physical environment. 


\subsection{Photoconductivity}

The origin of the photocurrent can be understood as follows: It has previously been shown that colloidal platinum can make electrical contact with the reducing end of photosystem I of photosynthesis. This contact was demonstrated by (1) the photocatalytic evolution of molecular hydrogen and (2) the observation of photocurrent in a sandwichlike photobioelectrochemical cell (Greenbaum 1989). Upon illumination, the platinum electrode in pressure contact with the platinized chloroplasts swung negative with respect to the silver-silver chloride electrode that was in pressure contact with the electrolyte-impregnated filter pad. No external bias was placed on the electrodes to force the direction of photocurrent flow.

A reasonable model for these results, based on the generally accepted structure of photosynthetic membranes (Marder and Barber 1989), is that colloidal platinum precipitated onto the external surface of the thylakoid membranes forms an isopotential surface whose distance is determined by the connectivity of the reticulated colloidal particles that are the metallic component of the chloroplast-metal composite matrix. This distance is a statistical parameter whose average value is determined by the nature of the platinum precipitation process. When platinum is precipitated, it does so in a nonspecific manner on the external surface of the thylakoid membrane. There is, however, an electrostatic between the negative charge of the hexachloroplatinate ions and the local positive charge of the lysine residues constituting part of the polypeptides of the photosystem I proteins (Colvert and Davis 1983).

The experimental system described in this work differs qualitatively from prior research performed with photosynthesis-based bioelectrochemical cells that utilized various organelles and components to generate photocurrents. Examples of prior research include chlorophyll liquid crystals (Aizawa et al 1978, 1979), pigmented bilayer membranes (Tien 1976), chloroplasts (Haehnel and Hochheimer 1979), chloroplast membranes (Allen et al 1974; Allen and Crane 1976; Allen 1977), algae (Ochiai et al 1980), and photosynthetic bacterial reaction centers (Janzen and Seibert 1980 ). For example, a photosystem II-enriched submembrane fraction in a photoelectrochemical cell operated in potentiostatic mode was used by Lemieux and Carpentier (1988) to generate photocurrents. The cell included artificial electron acceptors acting as charge transfer mediators between the photosynthetic membrane and the working electrode. Trissl and Kunze (1985) took another approach to generating and measuring photoelectric signals; they studied primary electrogenic reactions in chloroplasts probed by picosecond flash-induced dielectric polarization. Seibert and Kendall-Tobias (1982) measured photoelectrochemical properties of electrodes coated with photoactive-membrane vesicles isolated from photosynthetic bacteria. In their work, chromatophores isolated from the photosynthetic bacterium Rhodopseudomonas sphaeroides R-26 were prepared as a film on tin oxide electrodes, and the response to red light was examined in a liquid-junction photoelectrochemical cell. Alexandrowicz and Berns (1980) measured photovoltages in chloroplast extract bilayer membranes stimulated by micromolar amounts of oxidants and reductants.

The distinguishing feature of the work presented in this paper is that the planar composite matrix of precipitated platinum and chloroplast membranes is the conductive medium. That is to say, unlike chloroplast suspensions coupled to electrodes by redox-active mediators (there are no mediators in the preparation) or chloroplast preparations or films in close physical proximity to the electrodes, the composite 
photobioelectronic material itself is the photoconductive pathway. It was demonstrated that the concentration of the solution from which the platinum was precipitated directly affects the relative photoconductivity of the sample.

In conclusion, it has been demonstrated that electrical contact with the reducing end of photosystem I was achieved by precipitating colloidal platinum in the presence of isolated chloroplasts. The presence of the platinum had a significant effect on the photoconductivity of the metal-biological composite material. This work is technologically significant because the photosynthetic reaction centers are nanometer structures with picosecond switching times. This work demonstrates that the electron transport chain of photosynthesis can be electrically contacted and that the larger structural matrix of the platinized chloroplasts demonstrates enhanced photoconductivity.

\section{Acknowledgements}

I thank S L Blankinship and C V Tevault for technical support and D J Weaver and S A Hoglund for secretarial support. This research was supported by the US Department of Energy and Pittsburgh Energy Technology Center. Oak Ridge National Laboratory is managed by Martin Marietta Energy Systems. Inc., for the US Department of Energy under contract DE-AC-05-840R21400.

\section{References}

Aizawa M, Hirano M and Suzuki S 1978 J. Memb. Sci. 4251

Aizawa S, Hirano M and Suzuki S 1979 Electrochim. Acta 2489

Alexandrowicz G and Berns D S 1980 Photobiochem. Photobiophys. 1353

Allen M J 1977 in Living systems as energy converters (eds) R Buret, M J Allen and J P Massue (New York: North Holland) pp. 271-274

Allen M J and Crane A E 1976 Bioelectrochem. Bioenerg. 385

Allen M J, Curtis J A and Kerr M W 1974 Bioelectrochem. Bioenerg. 1408

Amesz J (ed.) 1987 Photosynthesis (Amsterdam: Elsevier)

Anderson J R 1975 Structure of metallic catalysts (New York: Academic Press)

Bishop N I, Frick M and Jones L W 1977 In Biological solar energy conversion (eds) A Mitsui, S Miyachi, A San Pietro and S Tamura (New York: Academic Press) pp. 3-22

Buchanan B B 1980 Annu. Rev. Plant. Physiol. 31341

Colvert K K and Davis D J 1983 Arch. Biochem. Biophys. 225936

Diner B and Mauzerall D 1973 Biochim. Biophys. Acta 305329

Forbush B and Kok B 1968 Biochim. Biophys. Acta 162243

Gaffron H and J Rubin 1942 J. Gen. Physiol. 26219

Gfeller R P and Gibbs M 1985 Plant Physiol. 77509

Greenbaum E 1979 Sol. Energy 23315

Greenbaum E 1984 Photobiochem. Photobiophys. 8323

Greenbaum E 1985 Science 2301373

Greenbaum E 1989 Bioelectrochem. Bioenerg. 21171

Hachnel W and Hochheimer H J 1979 Bioelectrochem. Bioenerg. 6563

Healey F P 1970 Plant Physiol 45153

Janzen A F and Seibert M 1980 Nature (London) 286584

Joliot P 1965 Biochem. Biophys. Acta 102116

Kaltwasser H, Stuart T S and Gaffron H 1969 Planta 89309

Lemieux S and Carpentier R 1988 J. Photochem. Photobiol. B2 221 
Lorimer G, Badger M R and Andrews T J 1976 Biochemistry 15529

Marder J B and Barber 1989 J. Plant, Cell Environ. 12595

Ochiai H, Shibata H, Sawa Y and Katoh T 1980 Proc. Natl. Acad. Sci. USA 772442

Peltier G, Ravenel J and Vermeglio A 1987 Biochim. Biophys. Acta 89383

Seibert M and Kendall-Tobias M W 1982 Biochim. Biophys. Acta 681504

Senger $\mathrm{H}$ and Bishop N I 1979 Planta 14553

Stiehl H H and Witt H T 1969 Z. Naturforsch. 2461588

Stuart T S and Gaffron H 1972 Plant Physiol. 50136

Tien H T 1976 Photochem. Photobiol. 2497

Trissl H W and Kunze U 1985 Biochim. Biophys. Acta 806, 136

Vermaas W F J and Govindjee 1981 Proc. Indian Natl. Sci. Acad. 847581

Walker D A 1980 Methods Enzymol. 6994

Ward B, Reeves M E and Greenbaum E 1985 Biotechnol. Bioeng. Symp. 15501 